\title{
CLASSICAL AND APPROXIMATE TAYLOR EXPANSIONS OF WEAKLY DIFFERENTIABLE FUNCTIONS
}

\author{
Paola Cavaliere and Andrea Cianchi \\ Università di Salerno, Dipartimento di Matematica \\ Via Giovanni Paolo II, 84084 Fisciano (SA), Italy; pcavaliere@unisa.it \\ Università di Firenze, Dipartimento di Matematica e Informatica "U. Dini" \\ Piazza Ghiberti 27, 50122 Firenze, Italy; andrea.cianchi@unifi.it
}

\begin{abstract}
The pointwise behavior of Sobolev-type functions, whose weak derivatives up to a given order belong to some rearrangement-invariant Banach function space, is investigated. We introduce a notion of approximate Taylor expansion in norm for these functions, which extends the usual definition of Taylor expansion in $L^{p}$-sense for standard Sobolev functions. An approximate Taylor expansion for functions in arbitrary-order Sobolev-type spaces, with sharp norm, is established. As a consequence, a characterization of those Sobolev-type spaces in which all functions admit a classical Taylor expansion is derived. In particular, this provides a higher-order version of a well-known result of Stein [27] on the differentiability of weakly differentiable functions. Applications of our results to customary classes of Sobolev-type spaces are also presented.
\end{abstract}

\section{Introduction}

The classical Lebesgue differentiation theorem asserts that if $u$ is any locally integrable function on an open set $\Omega$ in $\mathbf{R}^{n}$, with $n \geq 2$, then for almost every $x \in \Omega$

$$
\lim _{r \rightarrow 0^{+}} \frac{1}{|B(x, r)|} \int_{B(x, r)} u(y) d y
$$

exists, is finite, and agrees with $u(x)$. Here, $B(x, r)$ denotes the open ball of radius $r$ centered at $x$, and $|B(x, r)|$ its Lebesgue measure. The precise representative of $u$ is the function defined on $\Omega$ as the limit in (1.1) at those points $x$ for which such limit exists and is finite, and as 0 elsewhere. Hereafter, we assume that functions are precisely represented.

The additional assumption that $u$ belongs to $L_{\mathrm{loc}}^{p}(\Omega)$ for some $p \in[1, \infty[$ yields stronger information on the pointwise behaviour of $u$, namely

$$
\lim _{r \rightarrow 0^{+}}\|u-u(x)\|_{L^{p}(B(x, r))}^{\ominus}=0 \quad \text { for a.e. } x \in \Omega,
$$

where $\|\cdot\|_{L^{p}(B(x, r))}^{\ominus}$ stands for the averaged norm associated with $\|\cdot\|_{L^{p}(B(x, r))}$ and defined by

$$
\|u\|_{L^{p}(B(x, r))}^{\ominus}=\left(\frac{1}{|B(x, r)|} \int_{B(x, r)}|u(y)|^{p} d y\right)^{\frac{1}{p}} .
$$

doi:10.5186/aasfm.2014.3933

2010 Mathematics Subject Classification: Primary 46E35, 46E30.

Key words: Sobolev spaces, approximate differentiability, approximate Taylor expansion, rearrangement invariant spaces, Lorentz spaces, Orlicz spaces. 
In fact, equation (1.2) may be regarded as a 0-order Taylor expansion in norm-form for functions $u$ enjoying merely a locally $L^{p}$-integrability property on $\Omega$.

A parallel $m$-th order Taylor expansion in norm-form holds for functions endowed with weak derivatives up to the order $m \in \mathbf{N}$ in $L_{\text {loc }}^{p}(\Omega)$. Specifically, let $u \in W_{\text {loc }}^{m, p}(\Omega)$. If either $m \geq n$, or $p>1$ and $m p>n$, then $u$ admits a classical $m$-th order Taylor expansion at almost every point in $\Omega$, namely

$$
u(y)=T_{x}^{m}(u)(y)+o\left(|y-x|^{m}\right) \quad \text { as } y \rightarrow x,
$$

for a.e. $x \in \Omega$. When either $m p<n$, or $p>1$ and $m p=n$, the relevant expansion tells us that

$$
\lim _{r \rightarrow 0^{+}} \frac{1}{r^{m}}\left\|u-T_{x}^{m}(u)\right\|_{L^{q(B(x, r))}}^{\ominus}=0 \quad \text { for a.e. } x \in \Omega,
$$

with $q \leq \frac{n p}{n-m p}$ if $m p<n$, and $q<\infty$ if $p>1$ and $m p=n$ (see e.g. [28, Theorem 3.3.3, Chapter 3]). Here, $T_{x}^{m}(u)$ denotes the Taylor polynomial of order $m$ of $u$ centered at $x \in \Omega$, which is defined as

$$
T_{x}^{m}(u)(y)=\sum_{0 \leq|\alpha| \leq m} \frac{1}{\alpha !} D^{\alpha} u(x)(y-x)^{\alpha} \quad \text { for } y \in \mathbf{R}^{n},
$$

where $\alpha=\left(\alpha_{1}, \cdots, \alpha_{n}\right)$ is a multi-index of degree $|\alpha|=\sum_{i=1}^{n} \alpha_{i}, \alpha !=\alpha_{1} ! \cdots \alpha_{n}$ !, $(y-x)^{\alpha}=\left(y_{1}-x_{1}\right)^{\alpha_{1}} \cdots\left(y_{n}-x_{n}\right)^{\alpha_{n}}$, and $D^{\alpha}=D_{1}^{\alpha_{1}} \cdots D_{n}^{\alpha_{n}}$, with $D_{i}=\frac{\partial}{\partial x_{i}}$.

Enlarging the class of function spaces, and corresponding norms, which describe the integrability degree of functions and of their weak derivatives actually allows for more precise information on their pointwise differentiability properties. A wellknown contribution in this direction is due to Stein [27] (see also [17]), and tells us that if $u$ belongs to the Sobolev-type space of functions endowed with first-order weak derivatives which just belong to the Lorentz space $L_{\text {loc }}^{n, 1}(\Omega)$, then $u$ is differentiable a.e. in $\Omega$. Namely, $u$ fulfills (1.3) with $m=1$. This improves the parallel result in $W_{\text {loc }}^{1, p}(\Omega)$, with $p>n$, mentioned above, inasmuch as $L_{\text {loc }}^{p}(\Omega) \varsubsetneqq L_{\text {loc }}^{n, 1}(\Omega) \varsubsetneqq L_{\text {loc }}^{n}(\Omega)$ for $p>n$. In fact, the space $L_{\text {loc }}^{n, 1}(\Omega)$ is the optimal (largest possible) space enjoying this property in the class of all rearrangement-invariant (r.i., for short) spaces [10]. A precise definition of this class of spaces is given in the next section. Loosely speaking, membership of a measurable function $u$ to a (local) rearrangement-invariant space $X_{\text {loc }}(\Omega)$ only depends on its (local) integrability properties. Pointwise differentiability properties of functions whose weak derivatives belong to another customary class of r.i. spaces, the Orlicz spaces, have been investigated in $[2,12]$.

The present paper is aimed at providing a comprehensive approach to the problem of classical and approximate differentiability properties of functions in Sobolev-type spaces, of arbitrary order, built upon general rearrangement-invariant spaces. The analysis of these spaces has been the object of various contributions, especially in recent years, including $[11,14,15,16,18,20,21]$. However, apart from the specific results cited above in Lorentz and Orlicz spaces, this aspect of the theory seems to be still untouched in a general framework.

One difficulty which immediately arises in dealing with this topic is that, unlike Lebesgue norms, a general rearrangement-invariant norm need not have an integral form. A first problem to be faced is thus to find an appropriate counterpart $\|\cdot\|_{X(B(x, r))}^{\ominus}$ of the averaged norm $\|\cdot\|_{L^{p}(B(x, r))}^{\ominus}$ in (1.2). A notion of this kind is available in the 
literature $[1,13,22]$, and consists in defining the averaged norm in $X(B(x, r))$ as the ratio between the standard norm in $X(B(x, r))$ and the norm in $X(B(x, r))$ of the constant function 1 . Here, we instead propose and work with a different notion, which just amounts to replacing the Lebesgue measure $d x$ with the normalized Lebesgue measure $\frac{d x}{|B(x, r)|}$. Although these two definitions agree in some customary situations, they differ in general. The latter is more natural in a sense, and turns out to be better fitted for the problems under consideration.

It is however clear that an analogue of (1.2) for functions in $X_{\text {loc }}(\Omega)$, namely

$$
\lim _{r \rightarrow 0^{+}}\|u-u(x)\|_{X(B(x, r))}^{\ominus}=0 \quad \text { for a.e. } x \in \Omega,
$$

cannot hold without some additional assumption on the space $X_{\text {loc }}(\Omega)$, whatever reasonable definition of $\|\cdot\|_{X(B(x, r))}^{\otimes}$ is adopted. This can be verified, for instance, on taking $X_{\text {loc }}(\Omega)=L_{\text {loc }}^{\infty}(\Omega)$, a choice which makes (1.6) false.

Our main result can be summarized as follows. Given any space $X_{\text {loc }}(\Omega)$ fulfilling the 0-order approximate Taylor expansion (1.6), we show that an $m$-th order counterpart

$$
\lim _{r \rightarrow 0^{+}} \frac{1}{r^{m}}\left\|u-T_{x}^{m}(u)\right\|_{Y(B(x, r))}^{\ominus}=0 \quad \text { for a.e. } x \in \Omega
$$

holds for every $u$ from the Sobolev-type space $W^{m} X_{\text {loc }}(\Omega)$ of those functions whose weak derivatives up to the order $m$ belong to $X_{\text {loc }}(\Omega)$, and we characterize the optimal (i.e. the smallest possible) rearrangement-invariant space $Y_{\text {loc }}(\Omega)$ which is admissible in (1.7). This is the content of Theorem 3.1, Section 3. Such a result provides us with a unified framework for Taylor expansions of weakly differentiable functions of any order and, as a consequence, enables us to characterize the spaces $X_{\text {loc }}(\Omega)$ whose norm is sufficiently strong for the classical expansion (1.3) to hold for any $u \in W^{m} X_{\text {loc }}(\Omega)$ (Corollary 3.3, Section 3). In particular, we show that in the case when $m<n$ the Lorentz space $L_{\mathrm{loc}}^{\frac{n}{m}, 1}(\Omega)$ is the optimal-the largest possible-(local) r.i. space $X_{\text {loc }}(\Omega)$ which enjoys such property, thus providing a higher-order version of Stein's result.

A full characterization of the spaces $X_{\text {loc }}(\Omega)$ for which (1.6) holds is an issue of independent interest, and seems to be unknown. However, sufficient conditions for (1.6) to be true can be derived from results appearing in the literature, and can be applied to prove (1.6) for various families of spaces $X_{\text {loc }}(\Omega)$. For instance, we obtain an enhanced form of the classical result (1.4), where the $L^{q}$-norm is replaced with a somewhat stronger Lorentz, or Lorentz-Zygmund, norm. We refer to Section 3 for this conclusion, where applications to the case when $X_{\text {loc }}(\Omega)$ is either a Lorentz or an Orlicz space are also discussed.

\section{Background}

2.1. Rearrangement-invariant spaces. In this section we recall some definitions and basic properties of decreasing rearrangements and rearrangement-invariant function norms. For more details and proofs, we refer to [3, 19].

Let $E$ be a (non-negligible) Lebesgue measurable subset of $\mathbf{R}^{n}, n \geq 1$, of finite measure. We denote by $\chi_{E}$ the characteristic function of $E$, and by $|E|$ its Lebesgue measure. 
The Riesz space of all (equivalence classes of) extended real-valued measurable functions on $E$ is denoted by $L^{0}(E)$, and $L_{+}^{0}(E)=\left\{f \in L^{0}(E): f \geq 0\right\}$. The decreasing rearrangement of a function $f$ in $L^{0}(E)$ is the function $f^{*} \in L_{+}^{0}([0, \infty[)$ defined by

$$
f^{*}(s)=\sup \{t \geq 0:|\{x \in E:|f(x)|>t\}|>s\} \quad \text { for } s \in[0, \infty[.
$$

Clearly, $f^{*}(s)=0$ if $s \geq|E|$. The signed decreasing rearrangement of $f$ is the function $f^{o}:[0,|E|] \rightarrow[-\infty, \infty]$ given by

$$
f^{o}(s)=\sup \{t \in \mathbf{R}:|\{x \in E: f(x)>t\}|>s\} \quad \text { for } s \in[0,|E|] .
$$

Note that $\left(f^{o}\right)^{*}=f^{*}$. The Hardy-Littlewood inequality tells us that

$$
\|f g\|_{L^{1}(E)} \leq \int_{0}^{|E|} f^{*}(s) g^{*}(s) d s
$$

for every $f, g \in L^{0}(E)$. The function $\left.f^{* *}:\right] 0, \infty[\rightarrow[0, \infty]$ given by

$$
\left.f^{* *}(s)=\frac{1}{s} \int_{0}^{s} f^{*}(t) d t \quad \text { for } s \in\right] 0, \infty[
$$

is non-decreasing and satisfies $f^{*} \leq f^{* *}$. Moreover,

$$
(f+g)^{* *} \leq f^{* *}+g^{* *}
$$

for every $f, g \in L_{+}^{0}(E)$.

A rearrangement-invariant Banach (extended) function norm-an r.i. function norm, for short - is a functional $\|\cdot\|_{X(0,1)}: L_{+}^{0}(0,1) \rightarrow[0, \infty]$ such that

(N1) $\|f+g\|_{X(0,1)} \leq\|f\|_{X(0,1)}+\|g\|_{X(0,1)}$ for all $f, g \in L_{+}^{0}(0,1)$;

$\|\alpha f\|_{X(0,1)}=\alpha\|f\|_{X(0,1)}$ for all $\alpha \in\left[0, \infty\left[, f \in L_{+}^{0}(0,1)\right.\right.$

(here, and in what follows, we adhere the convention that $0 \cdot \infty=0$ );

$\|f\|_{X(0,1)}>0$ if $f$ does not vanish a.e.;

(N2) $\|f\|_{X(0,1)} \leq\|g\|_{X(0,1)}$ whenever $f \leq g$ a.e.;

(N3) $\sup _{k}\left\|f_{k}\right\|_{X(0,1)}=\|f\|_{X(0,1)}$ if $\left\{f_{k}\right\} \subset L_{+}^{0}(0,1)$ with $f_{k} \nearrow f$ a.e. in $] 0,1[$;

(N4) $\|1\|_{X(0,1)}<\infty$;

(N5) there is a positive constant $C$ such that $\|f\|_{L^{1}(0,1)} \leq C\|f\|_{X(0,1)}$ for all $f \in$ $L_{+}^{0}(0,1)$;

(N6) $\|f\|_{X(0,1)}=\|g\|_{X(0,1)}$ for all $f, g \in L_{+}^{0}(0,1)$ such that $f^{*}=g^{*}$. by

The associate function norm of $\|\cdot\|_{X(0,1)}$ is the r.i. function norm $\|\cdot\|_{X^{\prime}(0,1)}$ defined

$$
\|g\|_{X^{\prime}(0,1)}=\sup \left\{\|f g\|_{L^{1}(0,1)}: f \in L_{+}^{0}(0,1),\|f\|_{X(0,1)} \leq 1\right\} .
$$

Given $E$ as above and an r.i. function norm $\|\cdot\|_{X(0,1)}$, the space $X(E)$ is defined as the collection of all functions $f \in L^{0}(E)$ such that the quantity

$$
\|f\|_{X(E)}=\left\|f^{*}\right\|_{X(0,1)}
$$

is finite. This quantity defines a norm on $X(E)$, called an r.i. norm, which makes $X(E)$ a Banach space, called an r.i. space. The space $X(0,1)$ is called the representation space of $X(E)$. The r.i. space $X^{\prime}(E)$ built upon the function norm $\|\cdot\|_{X^{\prime}(0,1)}$ is called the associate space of $X(E)$. Let us notice that, although $E$ is required to have finite measure, it may exceed 1 . Thus, the norm $\|\cdot\|_{X(E)}$, defined by $(2.7)$, is 
just equivalent to (but possibly different from) more customary norms on $X(E)$. If $f \in L^{0}(E)$ and $g \in X(E)$ satisfy the inequality

$$
\left.f^{* *}(s) \leq g^{* *}(s) \text { for } s \in\right] 0, \infty[,
$$

then $f \in X(E)$ as well, and $\|f\|_{X(E)} \leq\|g\|_{X(E)}$ (see e.g. [3, Chapter 2, Proposition 3.6 and Theorem 4.6]). The following properties hold for any r.i. spaces $X(E)$ and $Y(E)$ :

$$
L^{\infty}(E) \hookrightarrow X(E) \hookrightarrow L^{1}(E), \text { where } \hookrightarrow \text { stands for a continuous embedding; }
$$

(2.10) $X(E)=X^{\prime \prime}(E)$;

(2.11) if $f \in X(E)$ and $g \in X^{\prime}(E)$, then $f g \in L^{1}(E)$ and the Hölder inequality $\|f g\|_{L^{1}(E)} \leq\|f\|_{X(E)}\|g\|_{X^{\prime}(E)}$ holds;

(2.12) $X(E) \hookrightarrow Y(E)$ if, and only if $Y^{\prime}(E) \hookrightarrow X^{\prime}(E)$ and the embedding constants are the same.

Now, define the functional

$$
\|f\|_{X(E)}^{\ominus}=\left\|f^{*}(|E| \cdot)\right\|_{X(0,1)}
$$

for $f \in X(E)$. This is another r.i. norm on $X(E)$, which - as a consequence of the boundedness of the dilation operator in r.i. spaces [3, Proposition 5.11] — is equivalent to $\|f\|_{X(E)}$ up to constants depending only on $|E|$. We will refer to the r.i. norm $(2.13)$ as the averaged norm of $\|\cdot\|_{X(E)}$, since

$$
\|f\|_{X(E)}^{\ominus}=\|f\|_{X\left(E, \frac{d x}{|E|}\right)} \quad \text { for } f \in X(E)
$$

where $X\left(E, \frac{d x}{|E|}\right)$ denotes the r.i. space, defined as above, save that rearrangements are defined with the Lebesgue measure replaced with the normalized Lebesgue measure $\frac{d x}{|E|}$. This averaged norm is closely related to a norm exploited in [26] to define maximal operators associated with general function norms.

Let us warn the reader that, in what follows, we shall frequently consider norms $\|f\|_{X(F)}$ and $\|f\|_{X(F)}^{\ominus}$, where $F$ is a measurable subset of the domain $E$ of $f$. They have to be interpreted as the norms defined as in (2.7) and (2.13), where $f$ is replaced with $f \chi_{F}$.

Note that

$$
\|1\|_{X(E)}^{\oslash}=\|1\|_{X(0,1)} .
$$

Also, if $f \in X(E)$ and $g \in X^{\prime}(E)$, then

$$
\frac{1}{|E|} \int_{E}|f(x) g(x)| d x \leq\|f\|_{X(E)}^{\ominus}\|g\|_{X^{\prime}(E)}^{\ominus} .
$$

We recall now the definition of some customary, and less standard, instances of r.i. function norms of use in our applications. In what follows, we set $p^{\prime}=\frac{p}{p-1}$ for $p \in[1, \infty]$, and adopt the convention that $1 / \infty=0$.

Prototypal examples of r.i. function norms are the classical Lebesgue norms. Indeed, $\|f\|_{L^{p}(0,1)}=\left\|f^{*}\right\|_{L^{p}(0,1)}$, if $p \in\left[1, \infty\left[\right.\right.$, and $\|f\|_{L^{\infty}(0,1)}=f^{*}\left(0^{+}\right)$. Note that, by (2.9), $L^{\infty}(E)$ and $L^{1}(E)$ are the smallest and the largest, respectively, r.i. spaces on 
$E$. Moreover, the averaged norms are just given by

$$
\|f\|_{L^{p}(E)}^{\ominus}= \begin{cases}\left(\frac{1}{|E|} \int_{E}|f(x)|^{p} d x\right)^{\frac{1}{p}} & \text { if } 1 \leq p<\infty \\ \|f\|_{L^{\infty}(E)} & \text { if } p=\infty .\end{cases}
$$

Let $p, q \in[1, \infty]$. Assume that either $1<p<\infty$ and $1 \leq q \leq \infty$, or $p=q=1$, or $p=q=\infty$. Then the functional defined as

$$
\|f\|_{L^{p, q(0,1)}}=\left\|t^{\frac{1}{p}-\frac{1}{q}} f^{*}(t)\right\|_{L^{q}(0,1)}
$$

for $f \in L_{+}^{0}(0,1)$, is equivalent to an r.i. function norm. The corresponding r.i. space is called a Lorentz space. One has that $L^{p, p}(E)=L^{p}(E)$ for every $p \in[1, \infty]$. If $1 \leq q \leq r \leq \infty$, then $L^{p, q}(E) \hookrightarrow L^{p, r}(E)$, and the two spaces agree if and only if $q=r$.

Assume now that either $1<p<\infty, 1 \leq q \leq \infty$ and $\alpha \in \mathbf{R}$, or $p=1, q=1$ and $\alpha \geq 0$, or $p=\infty, q=\infty$ and $\alpha \leq 0$, or $p=\infty, 1 \leq q<\infty$ and $\alpha+\frac{1}{q}<0$. Then also the functional given by

$$
\|f\|_{L^{p, q(\log L)^{\alpha}(0,1)}}=\left\|t^{\frac{1}{p}-\frac{1}{q}}\left(1+\log \frac{1}{t}\right)^{\alpha} f^{*}(t)\right\|_{L^{q}(0,1)}
$$

for $f \in L_{+}^{0}(0,1)$ is equivalent to an r.i. function norm. The r.i. space built upon this function norm is called a Lorentz-Zygmund space.

Let $A$ be a Young function, namely a left-continuous convex function from $[0, \infty[$ into $[0, \infty]$, which is neither identically equal to 0 , nor to $\infty$. Thus, $A$ has the form

$$
A(t)=\int_{0}^{t} a(s) d s \quad \text { for } t \geq 0
$$

for some (non-trivial) non-decreasing left-continuous function $a:[0, \infty[\rightarrow[0, \infty]$. The Luxemburg r.i. function norm associated with $A$ is defined as

$$
\|f\|_{L^{A}(0,1)}=\inf \left\{\lambda>0: \int_{0}^{1} A\left(\frac{f(t)}{\lambda}\right) d t \leq 1\right\}
$$

for $f \in L_{+}^{0}(0,1)$. The space $L^{A}(E)$ is called an Orlicz space. In particular, $L^{A}(E)=$ $L^{p}(E)$ if $A(t)=t^{p}$ for $p \in\left[1, \infty\left[\right.\right.$, and $L^{A}(E)=L^{\infty}(E)$ if $A(t)=\infty \chi_{11, \infty[}(t)$.

Recall that $A$ is said to satisfy the $\Delta_{2}$-condition near infinity if there exist positive constants $C$ and $t_{0}$ such that

$$
A(2 t) \leq C A(t) \quad \text { for } t \geq t_{0}
$$

moreover, $A$ is said to be equivalent near infinity to another Young function $B$ if there exist positive constants $c, C$ and $t_{0}$ such that

$$
A(c t) \leq B(t) \leq A(C t) \quad \text { for } t \geq t_{0} .
$$

Note that

$L^{A}(E)=L^{B}(E)$ up to equivalent norms if and only if $A$ is equivalent to $B$ near infinity.

Finally, assume that $A$ is a Young function and $q \in] 1, \infty[$. If

$$
\int^{\infty} \frac{A(t)}{t^{1+q}} d t<\infty
$$


then the r.i. function norm given by

$$
\|f\|_{L^{(q, A)}(0,1)}=\left\|t^{-\frac{1}{q}} f^{*}(t)\right\|_{L^{A}(0,1)}
$$

for $f \in L_{+}^{0}(0,1)$ will be called an Orlicz-Lorentz norm [8, Proposition 2.1]. The class of the Orlicz-Lorentz spaces encompasses (up to equivalent norms) the Orlicz spaces and various instances of Lorentz and Lorentz-Zygmund spaces.

2.2. Spaces of Sobolev-type. Let $\Omega$ be a non-empty open set in $\mathbf{R}^{n}, n \geq 2$, with $|\Omega|<\infty$, and let $\|\cdot\|_{X(0,1)}$ be an r.i. function norm. For each $m \in \mathbf{N}$, the $m$-th order Sobolev-type space $W^{m} X(\Omega)$ is the Banach space

$W^{m} X(\Omega)=\left\{u \in X(\Omega): u\right.$ is $m$-times weakly differentiable in $\Omega$, and $D^{\alpha} u \in X(\Omega)$ for every multi-index $\alpha$ such that $|\alpha| \leq m\}$

endowed with the norm

$$
\|u\|_{W^{m} X(\Omega)}=\sum_{k=0}^{m}\left\|\nabla^{k} u\right\|_{X(\Omega)} .
$$

Here, $\nabla^{k} u$ denotes the vector of all derivatives $D^{\alpha} u$, with $\alpha=\left(\alpha_{1}, \cdots, \alpha_{n}\right)$ such that $\sum_{i=1}^{n} \alpha_{i}=k$, and $\left\|\nabla^{k} u\right\|_{X(\Omega)}$ is an abridged notation for $\left\|\left|\nabla^{k} u\right|\right\|_{X(\Omega)}$, where $\left|\nabla^{k} u\right|$ is the Euclidean norm of $\nabla^{k} u$. Moreover, $\nabla^{0} u$ stands just for $u$, and $\nabla^{1} u$ will also simply be denoted by $\nabla u$.

The local Sobolev space $W^{m} X_{\text {loc }}(\Omega)$ is accordingly defined as

$$
W^{m} X_{\text {loc }}(\Omega)=\left\{u \in L^{0}(\Omega): u \in W^{m} X\left(\Omega^{\prime}\right) \text { for all open sets } \Omega^{\prime} \subset \subset \Omega\right\},
$$

where $\Omega$ is an arbitrary open set, not necessarily of finite measure, and the notation $\Omega^{\prime} \subset \subset \Omega$ means that the closure of $\Omega^{\prime}$ is a compact set contained in $\Omega$.

The optimal target r.i. function norm in Sobolev-type embeddings for $W^{m} X(\Omega)$ can be characterized as follows. For each $m \in \mathbf{N}$, we denote by $\|\cdot\|_{X_{m}(0,1)}$ the rearrangement-invariant function norm whose associate norm obeys

$$
\|f\|_{X_{m}^{\prime}(0,1)}=\left\|s^{-1+\frac{m}{n}} \int_{0}^{s} f^{*}(r) d r\right\|_{X^{\prime}(0,1)}
$$

for all $f \in L_{+}^{0}(0,1)$. By [20, Theorem A] (see also [11, Theorem 6.2]), if $\Omega$ is bounded and has a Lipschitz boundary, then

$$
W^{m} X(\Omega) \hookrightarrow X_{m}(\Omega),
$$

and the embedding constant depends only on $n, m$ and $\Omega$. Moreover, $X_{m}(\Omega)$ is optimal in (2.28) among all r.i. spaces, in the sense that, if $(2.28)$ holds with $X_{m}(\Omega)$ replaced with some other r.i. space $Y(\Omega)$, then

$$
X_{m}(\Omega) \hookrightarrow Y(\Omega),
$$

or, equivalently,

$$
X_{m}(0,1) \hookrightarrow Y(0,1)
$$




\section{Main results and applications}

An r.i. function norm $\|\cdot\|_{X(0,1)}$ will be said to fulfill the Lebesgue point property if, for every open set $\Omega$ in $\mathbf{R}^{n}$, with $n \geq 1$, and every $u \in X_{\text {loc }}(\Omega)$,

$$
\lim _{r \rightarrow 0^{+}}\|u-u(x)\|_{X(B(x, r))}^{\ominus}=0 \quad \text { for a.e. } x \in \Omega \text {. }
$$

Our main result is the following theorem.

Theorem 3.1. Let $\Omega$ be an open set in $\mathbf{R}^{n}$, with $n \geq 2$, and let $m \in \mathbf{N}$. Assume that $\|\cdot\|_{X(0,1)}$ is an r.i. function norm fulfilling the Lebesgue point property. If $u \in W^{m} X_{\mathrm{loc}}(\Omega)$, then

$$
\lim _{r \rightarrow 0^{+}} \frac{1}{r^{m}}\left\|u-T_{x}^{m}(u)\right\|_{X_{m}(B(x, r))}^{\ominus}=0 \quad \text { for a.e. } x \in \Omega,
$$

where $\|\cdot\|_{X_{m}(0,1)}$ is the optimal r.i. function norm in the Sobolev embedding associated with $\|\cdot\|_{X(0,1)}$ and $m$ as in (2.27).

As will be shown in Corollary 3.3 below, when $m \geq n$ the assumption that $\|\cdot\|_{X(0,1)}$ fulfills the Lebesgue point property in Theorem 3.1 is a posteriori immaterial. Indeed, for these values of $m$, property (3.1) implies that any function $u \in W^{m} X_{\text {loc }}(\Omega)$ has an approximate differential of order $m$ a.e. in $\Omega$, whatever $\|\cdot\|_{X(0,1)}$ is.

Remark 3.2. Assume that $\|\cdot\|_{X(0,1)}, m$, and $\Omega$ are as in Theorem 3.1, and that $\|\cdot\|_{Y(0,1)}$ is any r.i. function norm satisfying (2.29). If $u \in W^{m} X_{\text {loc }}(\Omega)$, then

$$
\lim _{r \rightarrow 0^{+}} \frac{1}{r^{m}}\left\|u-T_{x}^{m}(u)\right\|_{Y(B(x, r))}^{\ominus}=0 \quad \text { for a.e. } x \in \Omega .
$$

Theorem 3.1 enables us to characterize those r.i. function norms $\|\cdot\|_{X(0,1)}$ with the property that every function from the Sobolev-type space $W^{m} X_{\text {loc }}(\Omega)$ admits an $m$-th order differential a.e. in $\Omega$. Note that the Lebesgue point property of $\|\cdot\|_{X(0,1)}$ is not required in this result.

Corollary 3.3. Let $\|\cdot\|_{X(0,1)}$ be an r.i. function norm, and $m \in \mathbf{N}$. Let $\Omega$ be any open set in $\mathbf{R}^{n}$, with $n \geq 2$.

Case $m \geq n$. If $u \in W^{m} X_{\mathrm{loc}}(\Omega)$, then

$$
u(y)=T_{x}^{m}(u)(y)+o\left(|y-x|^{m}\right) \quad \text { as } y \rightarrow x,
$$

for a.e. $x \in \Omega$.

Case $1 \leq m<n$. The following facts are equivalent:

(i) each $u \in W^{m} X_{\text {loc }}(\Omega)$ fulfils (3.3) for a.e. $x \in \Omega$;

(ii) $\left\|s^{-1+\frac{m}{n}}\right\|_{X^{\prime}(0,1)}<\infty$;

(iii) $X(0,1) \hookrightarrow L^{\frac{n}{m}, 1}(0,1)$.

In particular, the Lorentz norm $\|\cdot\|_{L^{\frac{n}{m}, 1_{(0,1)}}}$ is the weakest r.i. function norm having the property that every function from the associated $m$-th order Sobolev space satisfies (3.3).

In the remaining part of this section, we present applications of Theorem 3.1 and Corollary 3.3 to customary classes of Sobolev-type spaces.

Classical Sobolev spaces. As a first consequence of Theorem 3.1 and Corollary 3.3, one can recover, and somewhat improve, via a unified approach, the standard 
results for the usual Sobolev space $W_{\mathrm{loc}}^{m, p}(\Omega)$ recalled in Section 1. Indeed, if either $p>1$ and $m p>n$, or $p=1$ and $m \geq n$, then condition (ii) of Corollary 3.3 is satisfied with $X(0,1)=L^{p}(0,1)$; hence any $u \in W_{\mathrm{loc}}^{m, p}(\Omega)$ fulfills (3.3). On the other hand, if $m p<n$, then $\left(L^{p}\right)_{m}(0,1)=L^{\frac{n p}{n-m p}, p}(0,1)$, up to equivalent norms; this conclusion can be derived from [18, Remark 5.13]; see also [11, Theorem 6.9] for a self-contained proof. Hence, by Theorem 3.1,

$$
\lim _{r \rightarrow 0^{+}} \frac{1}{r^{m}}\left\|u-T_{x}^{m}(u)\right\|_{L^{\frac{n p}{n-m p}, p}(B(x, r))}^{\ominus}=0 \quad \text { for a.e. } x \in \Omega .
$$

In the borderline case when $m p=n$ and $p>1$, one has that $\left(L^{p}\right)_{m}(0,1)=$ $L^{\infty, p}(\log L)^{-1}(0,1)$, up to equivalent norms ([4]; see also $\left.[18,10,11]\right)$, and hence, by Theorem 3.1,

$$
\lim _{r \rightarrow 0^{+}} \frac{1}{r^{m}}\left\|u-T_{x}^{m}(u)\right\|_{L^{\infty, p}(\log L)^{-1}(B(x, r))}^{\ominus}=0 \quad \text { for a.e. } x \in \Omega .
$$

Note that (3.4) and (3.5) actually enhance (1.4), inasmuch as the function norm $\|\cdot\|_{L^{\frac{n p}{n-m p}, p}(0,1)}$ is stronger than $\|\cdot\|_{L^{\frac{n p}{n-m p}(0,1)}}$, and $\|\cdot\|_{L^{\infty, p}(\log L)^{-1}(0,1)}$ is stronger than $\|\cdot\|_{L^{q}(0,1)}$ for every $q<\infty$.

Lorentz-Sobolev spaces. Properties (3.4) and (3.5) are special cases of more general results for Lorentz-Sobolev spaces, which can be deduced via Theorem 3.1 and Corollary 3.3.

Let us begin by observing that the Lorentz function norm $\|\cdot\|_{L^{p, q(0,1)}}$, defined by (2.18), enjoys the Lebesgue point property if $1 \leq q \leq p<\infty$. To verify this assertion, note that [27, equation (2) and Remark 2] tell us that if $\Omega$ is any open set in $\mathbf{R}^{n}, n \geq 2$, and $u \in L_{\mathrm{loc}}^{p, q}(\Omega)$ then

$$
\lim _{r \rightarrow 0^{+}} \frac{\|u-u(x)\|_{L^{p, q(B(x, r))}}}{\|1\|_{L^{p, q(B(x, r))}}}=0 \quad \text { for a.e. } x \in \Omega \text {. }
$$

On the other hand,

$$
\|u-u(x)\|_{L^{p, q}(B(x, r))}^{\ominus}=\left(\frac{p}{q}\right)^{1 / q} \frac{\|u-u(x)\|_{L^{p, q}(B(x, r))}}{\|1\|_{L^{p, q}(B(x, r))}}
$$

if $|B(x, r)| \leq 1$. The Lebesgue point property of $\|\cdot\|_{L^{p, q_{(0,1)}}}$ follows from (3.6) and (3.7).

Now let $u \in W^{m} L_{\mathrm{loc}}^{p, q}(\Omega)$, with $m \in \mathbf{N}$. By Corollary 3.3, $u$ admits an $m$-th order differential a.e. in $\Omega$ if either $m \geq n$, or $m<n$ and $m p>n$ and $q>1$, or $m p=n$ and $q=1$. Indeed, in each of these cases, we have that $L^{p, q}(0,1) \hookrightarrow L^{\frac{n}{m}, 1}(0,1)$. If none of the above conditions is satisfied, then one can show that

$$
\left(L^{p, q}\right)_{m}(0,1)= \begin{cases}L^{\frac{n p}{n-m p}, q}(0,1) & \text { if } m p<n, \\ L^{\infty, q}(\log L)^{-1}(0,1) & \text { if } m p=n \text { and } q>1,\end{cases}
$$

up to equivalent norms - see [18, Remark 5.13], or [11, Theorem 6.9]. Hence, by Theorem 3.1, if $1 \leq q \leq p$ and $m p<n$, then

$$
\lim _{r \rightarrow 0^{+}} \frac{1}{r^{m}}\left\|u-T_{x}^{m} u\right\|_{L^{\frac{n p}{n-m p}, q}(B(x, r))}^{\ominus}=0 \quad \text { for a.e. } x \in \Omega
$$


whereas if $1<q \leq p$ and $m p=n$

$$
\lim _{r \rightarrow 0^{+}} \frac{1}{r^{m}}\left\|u-T_{x}^{m}(u)\right\|_{L^{\infty, q}(\log L)^{-1}(B(x, r))}^{\ominus}=0 \quad \text { for a.e. } x \in \Omega .
$$

Orlicz-Sobolev spaces. Here, we focus the case when the r.i. function norm $\|\cdot\|_{X(0,1)}$ is a Luxemburg function norm $\|\cdot\|_{L^{A}(0,1)}$ associated with some Young function $A$. Let us preliminarily observe that such a function norm fulfills the Lebesgue point property provided that $A$ satisfies the $\Delta_{2}$-condition near infinity. In fact, by $[2$, Lemma 3.1] and such condition for $A$, one has that, if $\Omega$ is any open set in $\mathbf{R}^{n}$, $n \geq 1$, and $u \in L_{\text {loc }}^{A}(\Omega)$, then for a.e. $x \in \Omega$, and any $\sigma>0$, there exists $R>0$ such that

$$
\frac{1}{|B(x, r)|} \int_{B(x, r)} A\left(\frac{|u(y)-u(x)|}{\sigma}\right) d y \leq 1
$$

if $0<r<R$. Equation (3.10) entails that

$$
\|u-u(x)\|_{L^{A}(B(x, r))}^{\ominus} \leq \sigma
$$

and hence, owing to the arbitrariness of $\sigma$,

$$
\lim _{r \rightarrow 0^{+}}\|u-u(x)\|_{L^{A}(B(x, r))}^{\ominus}=0 \quad \text { for a.e. } x \in \Omega \text {. }
$$

Given $m \in \mathbf{N}$, we use the abridged notation $W_{\text {loc }}^{m, A}(\Omega)$ for the Orlicz-Sobolev space $W^{m} L_{\text {loc }}^{A}(\Omega)$ associated with the Young function $A$. Let $u \in W_{\mathrm{loc}}^{m, A}(\Omega)$. Then Corollary 3.3 tells us that $u$ has an $m$-th order differential a.e. in $\Omega$ if and only if either $m \geq n$, or $m<n$ and

$$
\int^{\infty}\left(\frac{t}{A(t)}\right)^{\frac{m}{n-m}} d t<\infty .
$$

Indeed, condition (3.11) is equivalent to condition (ii) of Corollary 3.3 when $X(0,1)=$ $L^{A}(0,1)$; this follows from [5, equation 3.10] and [7, Lemma 2.3].

Assume next that $1 \leq m<n$ and (3.11) fails, namely

$$
\int^{\infty}\left(\frac{t}{A(t)}\right)^{\frac{m}{n-m}} d t=\infty .
$$

Let us replace, if necessary, $A$ with another Young function, still denoted by $A$, which is equivalent to the original one near infinity, and such that

$$
\int_{0}\left(\frac{t}{A(t)}\right)^{\frac{m}{n-m}} d t<\infty .
$$

For instance, we may choose $A$ in such a way that $A(t)=t$ near 0 . Owing to (2.24), such a replacement leaves the space $W_{\mathrm{loc}}^{m, A}(\Omega)$ unchanged. Thanks to [8, Theorem 3.7], if $A$ is given by (2.20), then the optimal target space $\left(L^{A}\right)_{m}(0,1)$ for Orlicz-Sobolev embeddings associated with the Young function $A$ is the OrliczLorentz space $L^{\left(\frac{m}{n}, B\right)}(0,1)$, where $B$ is the (finite-valued) Young function defined by

$$
B(t)=\int_{0}^{t} b(r) d r \quad \text { for } t \geq 0
$$


and $b$ is the non-decreasing, left-continuous function in $[0, \infty[$ whose (generalized) left-continuous inverse is given by

$$
b^{-1}(s)=\left(\int_{a^{-1}(s)}^{\infty}\left(\int_{0}^{r}\left(\frac{1}{a(t)}\right)^{\frac{m}{n-m}} d t\right)^{-\frac{n}{m}} \frac{d r}{a(r)^{\frac{n}{n-m}}}\right)^{\frac{m}{m-n}} \text { for } s \geq 0 .
$$

Note that, since $A(t) \leq t a(t)$ for $t \geq 0$, condition (3.13) ensures that the integral $\int_{0}\left(\frac{1}{a(t)}\right)^{\frac{m}{n-m}} d t$ converges. Moreover, the Young function $B$ does fulfill condition (2.25) with $q=n / m$ [7, Proposition 2.2]. Thus, if $A$ satisfies the $\Delta_{2}$-condition near infinity, then, by Theorem 3.1,

$$
\lim _{r \rightarrow 0^{+}} \frac{1}{r^{m}}\left\|u-T_{x}^{m}(u)\right\|_{L^{\left(\frac{m}{n}, B\right)}(B(x, r))}^{\ominus}=0 \quad \text { for a.e. } x \in \Omega .
$$

A result in the spirit of (3.15) holds with the optimal r.i. norm replaced with an optimal Orlicz norm, when (3.12) is in force. Let $A_{\frac{n}{m}}$ be the Young function given by

$$
A_{\frac{n}{m}}(t)=A\left(H_{\frac{n}{m}}^{-1}(t)\right) \text { for } t \geq 0,
$$

where $H_{\frac{n}{m}}^{-1}$ is the generalized left-continuous inverse of the function $H_{\frac{n}{m}}$ obeying

$$
H_{\frac{n}{m}}(r)=\left(\int_{0}^{r}\left(\frac{t}{A(t)}\right)^{\frac{m}{n-m}} d t\right)^{\frac{n-m}{n}} \quad \text { for } r \geq 0 .
$$

Then

$$
W^{m, A}(\Omega) \hookrightarrow L^{A \frac{n}{m}}(\Omega)
$$

for every bounded open set $\Omega$ in $\mathbf{R}^{n}$ with a Lipschitz boundary, and $L^{A \frac{n}{m}}(\Omega)$ is optimal (i.e. smallest possible) among all Orlicz spaces [8, Theorem 3.1]. Hence, from Remark 3.2, we deduce that

$$
\lim _{r \rightarrow 0^{+}} \frac{1}{r^{m}}\left\|u-T_{x}^{m}(u)\right\|_{L}^{\ominus}{ }_{\frac{n}{m}(B(x, r))}=0 \quad \text { for a.e. } x \in \Omega \text {. }
$$

Let us specialize the results discussed above to the case when $A$ is a Young function equivalent to $t^{p} \log ^{\alpha}(1+t)$ near infinity, where either $p>1$ and $\alpha \in \mathbf{R}$, or $p=1$ and $\alpha \geq 0$. A real number $\alpha$ satisfying this assumption will be called admissible in what follows. Clearly, $A$ satisfies the $\Delta_{2}$-condition near infinity, and hence the Orlicz norm $\|\cdot\|_{L^{A}(0,1)}$ enjoys the Lebesgue point property. We may clearly just focus the case when $m<n$. The function $A$ fulfils (3.11) if either $p>\frac{n}{m}$, or $p=\frac{n}{m}$ and $\alpha>\frac{n}{m}-1$. In both cases, Corollary 3.3 tells us that each function $u \in W_{\text {loc }}^{m, A}(\Omega)$ has a $m$-th order differential a.e. in $\Omega$. When (3.11) fails, one has

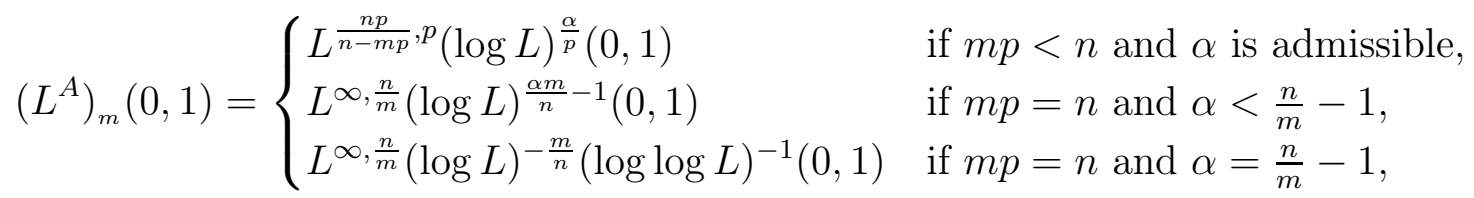

up to equivalent norms [8, Example 3.10]. The last space is a generalized LorentzZygmund space associated with the r.i. function norm given by

$$
\|f\|_{L^{\infty, \frac{n}{m}(\log L)^{-\frac{m}{n}}(\log \log L)^{-1}(0,1)}}=\left\|s^{-\frac{m}{n}}\left(1+\log \left(\frac{1}{s}\right)\right)^{-\frac{m}{n}}\left(1+\log \left(1+\log \left(\frac{1}{s}\right)\right)\right)^{-1} f^{*}(s)\right\|_{L \frac{n}{m}(0,1)}
$$


for $f \in L_{+}^{0}(0,1)$.

Now, let $u \in W_{\text {loc }}^{m, A}(\Omega)$. By Theorem 3.1, if $m p<n$ and $\alpha$ is any admissible number, then

$$
\lim _{r \rightarrow 0^{+}} \frac{1}{r^{m}}\left\|u-T_{x}^{m}(u)\right\|_{L^{\frac{n p}{n-m p}, p}(\log L)^{\frac{\alpha}{p}}(B(x, r))}^{\otimes}=0 \quad \text { for a.e. } x \in \Omega .
$$

When $m p=n$, if $\alpha<\frac{n}{m}-1$, then

$$
\lim _{r \rightarrow 0^{+}} \frac{1}{r^{m}}\left\|u-T_{x}^{m}(u)\right\|_{L^{\infty, \frac{n}{m}(\log L)^{\frac{\alpha m}{n}-1}(B(x, r))}}^{\otimes}=0 \quad \text { for a.e. } x \in \Omega,
$$

whereas if $\alpha=\frac{n}{m}-1$, then

$$
\lim _{r \rightarrow 0^{+}} \frac{1}{r^{m}}\left\|u-T_{x}^{m}(u)\right\|_{L^{\infty, \frac{n}{m}(\log L)^{-\frac{m}{n}}(\log \log L)^{-1}(B(x, r))}}^{\otimes}=0 \quad \text { for a.e. } x \in \Omega .
$$

Finally, $u$ fulfills (3.16), where

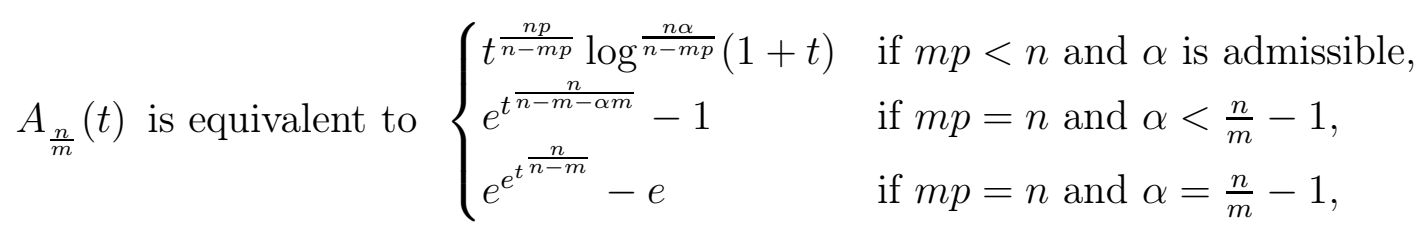

near infinity.

\section{Proofs of the main results}

We preliminarily state and prove some auxiliary lemmas.

Lemma 4.1. Let $n \in \mathbf{N}$, with $n \geq 2$, and let $x \in \mathbf{R}^{n}$. Let $\|\cdot\|_{X(0,1)}$ be an r.i. function norm. Then there exists a positive constant $C=C(n)$ such that

$$
\left\|u-u_{B(x, 1)}\right\|_{X(B(x, 1))}^{\ominus} \leq C\|\nabla u\|_{X(B(x, 1))}^{\ominus}
$$

for every $u \in W^{1} X(B(x, 1))$. Here, $u_{B(x, 1)}=\frac{1}{|B(x, 1)|} \int_{B(x, 1)} u(y) d y$.

Proof. We may assume, without loss of generality, that $x=0$. Let $u \in$ $W^{1} X(B(0,1))$. In particular, by $(2.9), u \in W^{1,1}(B(0,1))$ as well. Then [9, Lemma 6.6 and (6.30)] tell us that its signed decreasing rearrangement $u^{o}$ is locally absolutely continuous in $] 0, \omega_{n}\left[\right.$, where $\omega_{n}=|B(0,1)|$, and

$$
\left.u^{\circ}(s)-u_{B(0,1)}=\int_{0}^{\omega_{n}}\left(\chi_{\left(s, \omega_{n}\right)}(t)-\frac{t}{\omega_{n}}\right)\left(-u^{\circ}\right)^{\prime}(t) d t \quad \text { for } s \in\right] 0, \omega_{n}[
$$

Moreover, coupling [10, Lemma 4.1 and (3.5)] with property (N2) of r.i. function norms implies that there exists a positive constant $C^{\prime}=C^{\prime}(n)$ such that

$$
\left\|\min \left\{t, \omega_{n}-t\right\}^{\frac{1}{n^{\prime}}}\left(-u^{\circ}\right)^{\prime}(t)\right\|_{X\left(0, \omega_{n}\right)}^{\ominus} \leq C^{\prime}\|\nabla u\|_{X(B(0,1))}^{\ominus} .
$$

Consider the linear operator $T$ defined, for $\varphi \in L^{1}\left(0, \omega_{n}\right)$, by

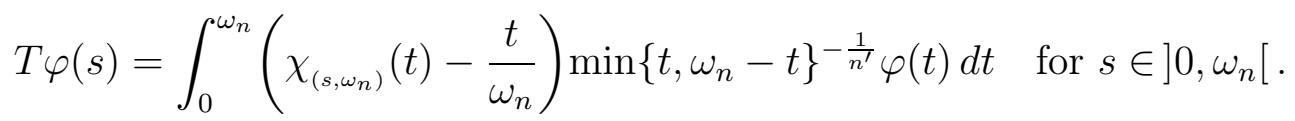

It is easy to check that $T$ is bounded on $L^{1}\left(0, \omega_{n}\right)$, and on $L^{\infty}\left(0, \omega_{n}\right)$. In particular,

$$
\|T \varphi\|_{L^{1}\left(0, \omega_{n}\right)}^{\ominus} \leq 2 \omega_{n}^{1 / n}\|\varphi\|_{L^{1}\left(0, \omega_{n}\right)}^{\ominus}
$$


for $\varphi \in L^{1}\left(0, \omega_{n}\right)$, and

$$
\|T \varphi\|_{L^{\infty}\left(0, \omega_{n}\right)}^{\ominus} \leq 2 n \omega_{n}^{1 / n}\|\varphi\|_{L^{\infty}\left(0, \omega_{n}\right)}^{\ominus}
$$

for $\varphi \in L^{\infty}\left(0, \omega_{n}\right)$. An application of an interpolation theorem of Calderón [3, Chapter 3, Theorem 2.12] implies that $T$ is bounded on $X\left(0, \omega_{n}\right)$, and

$$
\|T \varphi\|_{X\left(0, \omega_{n}\right)}^{\ominus} \leq 2 n \omega_{n}^{1 / n}\|\varphi\|_{X\left(0, \omega_{n}\right)}^{\ominus}
$$

for $\varphi \in X\left(0, \omega_{n}\right)$. From (4.2), (4.4) and (4.3), one concludes that

$$
\left\|u-u_{B(0,1)}\right\|_{X(B(0,1))}^{\ominus}=\left\|u^{\circ}-u_{B(0,1)}\right\|_{X\left(0, \omega_{n}\right)}^{\ominus} \leq 2 n \omega_{n} C^{\prime}\|\nabla u\|_{X(B(0,1))}^{\ominus}
$$

whence (4.1) follows.

Lemma 4.2. Let $n \in \mathbf{N}$, with $n \geq 2$, and let $x \in \mathbf{R}^{n}$ and $r>0$. Let $\|\cdot\|_{X(0,1)}$ be an r.i. function norm, and let $\|\cdot\|_{X_{1}(0,1)}$ be the optimal target r.i. function norm in the first-order Sobolev embedding associated with $\|\cdot\|_{X(0,1)}$ as in (2.27). Then there exists a constant $C_{1}=C_{1}(n)$ such that

$$
\left\|u-u_{B(x, r)}\right\|_{X_{1}(B(x, r))}^{\ominus} \leq C_{1} r\|\nabla u\|_{X(B(x, r))}^{\ominus}
$$

for every $u \in W^{1} X(B(x, r))$. Hence, in particular, if $\|\cdot\|_{Y(0,1)}$ is any r.i. function norm satisfying (2.29) with $m=1$, then there exists a constant $C_{2}=C_{2}(n, Y)$ such that

$$
\left\|u-u_{B(x, r)}\right\|_{Y(B(x, r))}^{\ominus} \leq C_{2} r\|\nabla u\|_{X(B(x, r))}^{\ominus}
$$

for every $u \in W^{1} X(B(x, r))$.

Proof. We may assume, without loss of generality, that $x=0$. Let $u \in$ $W^{1} X(B(0, r))$, and define $w: B(0,1) \rightarrow \mathbf{R}$ by $w(y)=u(r y)$ for $y \in B(0,1)$. Note that $w \in W^{1} X(B(0,1))$. Then $(2.28)$ with $m=1$, and Lemma 4.1 , imply that

$$
\begin{aligned}
\left\|w-w_{B(0,1)}\right\|_{X_{1}(B(0,1))}^{\ominus} & \leq C\left(\left\|w-w_{B(0,1)}\right\|_{X(B(0,1))}^{\ominus}+\|\nabla w\|_{X(B(0,1))}^{\ominus}\right) \\
& \leq C\left(C^{\prime}\|\nabla w\|_{X(B(0,1))}^{\ominus}+\|\nabla w\|_{X(B(0,1))}^{\ominus}\right) \\
& =C\left(C^{\prime}+1\right)\|\nabla w\|_{X(B(0,1))}^{\ominus}
\end{aligned}
$$

for some positive constants $C$ and $C^{\prime}$ depending only on $n$.

Computations show that

$$
\left.w^{o}\left(\omega_{n} s\right)=u^{o}\left(\omega_{n} r^{n} s\right) \quad \text { for } s \in\right] 0,1\left[, \quad \text { and } \quad w_{B(0,1)}=u_{B(0, r)} .\right.
$$

Consequently,

$$
\begin{aligned}
\left(w-w_{B(0,1)}\right)^{*}\left(\omega_{n} s\right) & =\left(w^{o}-w_{B(0,1)}\right)^{*}\left(\omega_{n} s\right)=\left(u^{o}-u_{B(0, r)}\right)^{*}\left(\omega_{n} r^{n} s\right) \\
& \left.=\left(u-u_{B(0, r)}\right)^{*}\left(\omega_{n} r^{n} s\right) \quad \text { for } s \in\right] 0,1[.
\end{aligned}
$$

Thus,

$$
\begin{aligned}
\left\|w-w_{B(0,1)}\right\|_{X_{1}(B(0,1))}^{\ominus} & =\left\|\left(w-w_{B(0,1)}\right)^{*}\left(\omega_{n} \cdot\right)\right\|_{X_{1}(0,1)} \\
& =\left\|\left(u-u_{B(0, r)}\right)^{*}\left(\omega_{n} r^{n} \cdot\right)\right\|_{X_{1}(0,1)}=\left\|u-u_{B(0, r)}\right\|_{X_{1}(B(0, r))}^{\ominus} .
\end{aligned}
$$

Next,

$$
\left.|\nabla w|^{*}\left(\omega_{n} s\right)=r|\nabla u|^{*}\left(\omega_{n} r^{n} s\right) \quad \text { for } s \in\right] 0,1[
$$


whence

$$
\|\nabla w\|_{X(B(0,1))}^{\ominus}=\left\||\nabla w|^{*}\left(\omega_{n} \cdot\right)\right\|_{X(0,1)}=r\left\||\nabla u|^{*}\left(\omega_{n} r^{n} \cdot\right)\right\|_{X(0,1)}=r\|\nabla u\|_{X(B(0, r))}^{\ominus}
$$

Combining (4.7), (4.8) and (4.9) yields

$$
\frac{1}{r}\left\|u-u_{B(0, r)}\right\|_{X_{1}(B(0, r))}^{\ominus} \leq C_{1}\|\nabla u\|_{X(B(0, r))}^{\ominus}
$$

with $C_{1}=C\left(C^{\prime}+1\right)$.

Lemma 4.3. Let $\|\cdot\|_{X(0,1)}$ be an r.i. function norm, and let $\Omega$ be an open set in $\mathbf{R}^{n}$, with $n \geq 2$. If $u \in W^{1} X_{\text {loc }}(\Omega)$, then

$$
\frac{1}{r}\|u-u(x)\|_{X(B(x, r))}^{\ominus} \leq \sup _{\rho \in[0, r]}\|\nabla u\|_{X(B(x, \rho))}^{\ominus} \quad \text { for a.e. } x \in \Omega,
$$

for every $r>0$ such that $B(x, r) \subset \subset \Omega$.

Proof. Let $u \in W^{1} X_{\text {loc }}(\Omega)$. Then, by (2.9), $u \in W_{\text {loc }}^{1,1}(\Omega)$. A standard result in the theory of Sobolev spaces tells us that, for a.e. $x \in \Omega$ and every $r>0$ such that $B(x, r) \subset \subset \Omega$, the function $[0,1] \ni t \mapsto u(x+t(y-x))$ is absolutely continuous for a.e. $y \in B(x, r)$, and

$$
\frac{d}{d t} u(x+t(y-x))=\nabla u(x+t(y-x)) \cdot(y-x) \quad \text { for a.e. } t \in[0,1],
$$

where "." stands for scalar product in $\mathbf{R}^{n}$. Hence,

$$
|u(y)-u(x)| \leq \int_{0}^{1}|\nabla u(x+t(y-x))||y-x| d t \quad \text { for a.e. } y \in B(x, r) .
$$

An application of the integral Minkowski inequality then yields

$$
\begin{aligned}
\frac{1}{r}\|u-u(x)\|_{X(B(x, r))}^{\ominus} & \leq\left\|\int_{0}^{1} \frac{1}{r}|\nabla u(x+t(y-x))||y-x| d t\right\|_{X(B(x, r))}^{\ominus} \\
& \leq \int_{0}^{1}\left\|\frac{|\cdot-x|}{r}|\nabla u(x+t(\cdot-x))|\right\|_{X(B(x, r))}^{\ominus} d t \\
& \leq \int_{0}^{1}\|\nabla u(x+t(\cdot-x))\|_{X(B(x, r))}^{\ominus} d t \\
& =\int_{0}^{1}\|\nabla u\|_{X(B(x, r t))}^{\ominus} d t \leq \sup _{\rho \in[0, r]}\|\nabla u\|_{X(B(x, \rho))}^{\ominus},
\end{aligned}
$$

namely (4.10).

We are now in a position to accomplish the proof of Theorem 3.1.

Proof of Theorem 3.1. Let $u \in W^{m} X_{\text {loc }}(\Omega)$. We claim that, for each $k \in \mathbf{N}$, with $k \leq m$, there exists a constant $C=C(n, k, X)$ such that

$$
\frac{1}{r^{k}}\left\|u-T_{x}^{k}(u)\right\|_{X_{k}(B(x, r))}^{\ominus} \leq C \sup _{\rho \in[0, r]}\left\|\nabla^{k} u-\nabla^{k} u(x)\right\|_{X(B(x, \rho))}^{\ominus} \quad \text { for a.e. } x \in \Omega
$$

for every $r>0$ such that $B(x, r) \subset \subset \Omega$. 
Consider first the case when $k=1$. For each $x \in \Omega$, the function $v=\frac{1}{r}\left(u-T_{x}^{1}(u)\right)$ belongs to $W^{1} X_{\text {loc }}(\Omega)$. Thus, owing to Lemma 4.2 , for any ball $B(x, r) \subset \subset \Omega$

$$
\left\|v-v_{B(x, r)}\right\|_{X_{1}(B(x, r))}^{\ominus} \leq C_{1} r\|\nabla v\|_{X(B(x, r))}^{\ominus}
$$

for some positive constant $C_{1}=C_{1}(n)$. By (4.12) and (2.16),

$$
\begin{aligned}
\|v\|_{X_{1}(B(x, r))}^{\ominus} & \leq\left\|v-v_{B(x, r)}\right\|_{X_{1}(B(x, r))}^{\ominus}+\left\|v_{B(x, r)}\right\|_{X_{1}(B(x, r))}^{\ominus} \\
& \leq C_{1} r\|\nabla v\|_{X(B(x, r))}^{\ominus}+\left|v_{B(x, r)}\right|\|1\|_{X_{1}(B(x, r))}^{\ominus}\|\|_{X(B(x, r))}\|1\|_{X^{\prime}(B(x, r))}^{\ominus}\|1\|_{X_{1}(B(x, r))}^{\ominus} \\
& \leq C_{1} r\|\nabla v\|_{X(B(x, r))}^{\ominus}+\|v\|_{X(B(x, r))}^{\ominus}
\end{aligned}
$$

where, by (2.15), $C^{\prime}$ is independent of $r$, and equals $\|1\|_{X^{\prime}(0,1)}\|1\|_{X_{1}(0,1)}$. Consequently,

$$
\frac{1}{r}\left\|u-T_{x}^{1}(u)\right\|_{X_{1}(B(x, r))}^{\ominus} \leq C_{1}\|\nabla u-\nabla u(x)\|_{X(B(x, r))}^{\ominus}+\frac{C^{\prime}}{r}\left\|u-T_{x}^{1}(u)\right\|_{X(B(x, r))}^{\ominus}
$$

for each $x \in \Omega$, and every $r>0$ such that $B(x, r) \subset \subset \Omega$. Applying Lemma 4.3, with $u$ replaced with $u-T_{x}^{1}(u)$, tells us that

$$
\frac{1}{r}\left\|u-T_{x}^{1}(u)\right\|_{X(B(x, r))}^{\ominus} \leq \sup _{\rho \in[0, r]}\|\nabla u-\nabla u(x)\|_{X(B(x, \rho))}^{\ominus} \quad \text { for a.e. } x \in \Omega,
$$

for every $r>0$ such that $B(x, r) \subset \subset \Omega$. Combining (4.14) and (4.15) thus yields

$$
\begin{aligned}
\frac{1}{r}\left\|u-T_{x}^{1}(u)\right\|_{X_{1}(B(x, r))}^{\ominus} & \leq C_{1}\|\nabla u-\nabla u(x)\|_{X(B(x, r))}^{\ominus}+C^{\prime} \sup _{\rho \in[0, r]}\|\nabla u-\nabla u(x)\|_{X(B(x, \rho))}^{\ominus} \\
& \leq\left(C_{1}+C^{\prime}\right) \sup _{\rho \in[0, r]}\|\nabla u-\nabla u(x)\|_{X(B(x, \rho))}^{\ominus},
\end{aligned}
$$

namely, inequality (4.11) with $k=1$.

We now argue by finite induction, and assume that (4.11) holds for some $k \in \mathbf{N}$, with $k<m$. Since $u \in W^{k+1} X_{\text {loc }}(\Omega)$, one has that $u-T_{x}^{k+1}(u) \in W^{k+1} X_{\text {loc }}(\Omega)$ for each $x \in \Omega$. Hence, $u-T_{x}^{k+1}(u) \in W^{1}\left(X_{k}\right)_{\text {loc }}(\Omega)$. By [11, Theorem 5.7],

$$
\left(X_{k+1}\right)_{\mathrm{loc}}(\Omega)=\left(\left(X_{k}\right)_{1}\right)_{\mathrm{loc}}(\Omega) \text {. }
$$

From (4.13) with $v$ and $\|\cdot\|_{X(0,1)}$ replaced with $u-T_{x}^{k+1}(u)$ and $\|\cdot\|_{X_{k}(0,1)}$, respectively, and (4.16), one can deduce that

$$
\begin{aligned}
& \frac{1}{r^{k+1}}\left\|u-T_{x}^{k+1}(u)\right\|_{X_{k+1}(B(x, r))}^{\ominus} \\
& \leq \frac{C_{1}}{r^{k}}\left\|\nabla\left(u-T_{x}^{k+1}(u)\right)\right\|_{X_{k}(B(x, r))}^{\ominus}+\frac{C^{\prime}}{r^{k+1}}\left\|u-T_{x}^{k+1}(u)\right\|_{X_{k}(B(x, r))}^{\ominus} \\
& =\frac{C_{1}}{r^{k}}\left\|\nabla u-T_{x}^{k}(\nabla u)\right\|_{X_{k}(B(x, r))}^{\ominus}+\frac{C^{\prime}}{r^{k+1}}\left\|u-T_{x}^{k+1}(u)\right\|_{X_{k}(B(x, r))}^{\ominus}
\end{aligned}
$$

for each $x \in \Omega$, and every $r>0$ such that $B(x, r) \subset \subset \Omega$.

By the induction assumption, one can apply (4.11), with $u$ replaced with each partial derivative $u_{x_{i}}$, and obtain that there exists a positive constant $C=C(n, k, X)$ such that

$$
\frac{1}{r^{k}}\left\|\nabla u-T_{x}^{k}(\nabla u)\right\|_{X_{k}(B(x, r))}^{\ominus} \leq C \sup _{\rho \in[0, r]}\left\|\nabla^{k+1} u-\nabla^{k+1} u(x)\right\|_{X(B(x, \rho))}^{\ominus}
$$


for a.e. $x \in \Omega$, for every $r>0$ such that $B(x, r) \subset \subset \Omega$. Moreover, Lemma 4.3 applied with $\|\cdot\|_{X(0,1)}$ and $u$ replaced with $\|\cdot\|_{X_{k}(0,1)}$ and $u-T_{x}^{k+1}(u)$, respectively, yields

$$
\frac{1}{r}\left\|u-T_{x}^{k+1}(u)\right\|_{X_{k}(B(x, r))}^{\ominus} \leq \sup _{\rho \in[0, r]}\left\|\nabla u-T_{x}^{k}(\nabla u)\right\|_{X_{k}(B(x, \rho))}^{\ominus} .
$$

Therefore, owing to (4.18),

$$
\begin{aligned}
\frac{1}{r^{k+1}}\left\|u-T_{x}^{k+1}(u)\right\|_{X_{k}(B(x, r))}^{\ominus} & \leq \frac{1}{r^{k}} \sup _{\rho \in[0, r]}\left\|\nabla u-T_{x}^{k}(\nabla u)\right\|_{X_{k}(B(x, \rho))}^{\ominus} \\
& \leq \sup _{\rho \in[0, r]} \frac{1}{\rho^{k}}\left\|\nabla u-T_{x}^{k}(\nabla u)\right\|_{X_{k}(B(x, \rho))}^{\ominus} \\
& \leq C \sup _{\rho \in[0, r]} \sup _{\sigma \in[0, \rho]}\left\|\nabla^{k+1} u-\nabla^{k+1} u(x)\right\|_{X(B(x, \sigma))}^{\ominus} \\
& =C \sup _{\varrho \in[0, r]}\left\|\nabla^{k+1} u-\nabla^{k+1} u(x)\right\|_{X(B(x, \varrho))}^{\ominus}
\end{aligned}
$$

for a.e. $x \in \Omega$, and every $r>0$ such that $B(x, r) \subset \subset \Omega$. Combining (4.17)-(4.19) entails that (4.11) holds with $k+1$ in place of $k$. Estimate (4.11) is thus fully established for every $k \leq m$.

Equation (3.1) now follows from (4.11), with $k=m$, via the Lebesgue point property of $\|\cdot\|_{X(0,1)}$.

We conclude with a proof of Corollary 3.3.

Proof of Corollary 3.3. Case $m \geq n$. Let $u \in W^{m} X_{\text {loc }}(\Omega)$. Hence, by (2.9), $u \in W^{m} L_{\text {loc }}^{1}(\Omega)$. The Lebesgue norm $\|\cdot\|_{L^{1}(0,1)}$ enjoys the Lebesgue point property. Moreover, since $m \geq n$, one has that $\left(L^{1}\right)_{m}(0,1)=L^{\infty}(0,1)$ by a superlimiting case of the standard Sobolev embedding theorem [23, Theorem 1.4.5]. Also, the function $u$ is continuous. Theorem 3.1 thus tells us that

$$
\lim _{r \rightarrow 0^{+}} \frac{1}{r^{m}}\left\|u-T_{x}^{m}(u)\right\|_{L^{\infty}(B(x, r))}=0 \quad \text { for a.e. } x \in \Omega \text {. }
$$

Hence,

$$
\lim _{y \rightarrow x} \frac{\left|u(y)-T_{x}^{m}(u)(y)\right|}{|y-x|^{m}}=0 \quad \text { for a.e. } x \in \Omega
$$

namely (3.3).

Case $m<n$. The equivalence of conditions (iii) and (ii) is a consequence of the fact that, by (2.6), and (2.18) with $p=\frac{m}{n}$ and $q=1$,

$$
\left\|s^{\frac{m-n}{n}}\right\|_{X^{\prime}(0,1)}=\sup _{f \in X(0,1)} \frac{\|f\|_{L^{\frac{n}{m}, 1}(0,1)}}{\|f\|_{X(0,1)}} .
$$

To verify that (iii) implies (i), fix any $u \in W^{m} X_{\text {loc }}(\Omega)$. Owing to (iii), one has that $u \in W^{m} L_{\text {loc }}^{\frac{n}{m}, 1}(\Omega)$. The Lorentz norm $\|\cdot\|_{L^{\frac{n}{m}, 1}(0,1)}$ enjoys the Lebesgue point property, as observed in Section 3. One has that $\left(L^{\frac{n}{m}, 1}\right)_{m}(0,1)=L^{\infty}(0,1)[24,25]$. Furthermore, any function $u \in W^{m} L_{\text {loc }}^{\frac{n}{m}, 1}(\Omega)$ is continuous, as can be shown by the Sobolev embedding theorem in Lorentz spaces [25], combined with the first-order result of [27] (see also [12, Theorem 3.1]). Thus, by Theorem 3.1, equation (4.20) holds, and (3.3) follows as above. Property (i) is thus proved. 
We finally show that (i) implies (iii). Assume, by contradiction, that (iii) fails. Then the inclusion $X(0,1) \subset L^{\frac{n}{m}, 1}(0,1)$ fails as well [3, Theorem 1.8], namely, there exists a function $f \in X(0,1) \backslash L^{\frac{n}{m}, 1}(0,1)$. Without loss of generality, we may assume that $0 \in \Omega$, and let $R>0$ be such that $B(0, R) \subset \subset \Omega$. Define $u: \Omega \rightarrow[0, \infty[$ as

$$
u(x)= \begin{cases}\int_{\omega_{n}|x|^{n}}^{\omega_{n} f^{n}} f^{*}(t) t^{-m+\frac{m}{n}}\left(t-\omega_{n}|x|^{n}\right)^{m-1} d t & \text { if } x \in B(0, R), \\ 0 & \text { if } x \in \Omega \backslash B(0, R) .\end{cases}
$$

Note that $u$ is radially decreasing and $m$-times weakly-differentiable. Moreover, by [20, proof of Theorem A], one has that $u \in W^{m} X_{\text {loc }}(\Omega)$, and

$$
\|u\|_{W^{m_{X}(B)}} \leq C\|f\|_{X(0,1)}
$$

for some positive constant $C=C(m, n, R)$. On the other hand, $u \notin L_{\mathrm{loc}}^{\infty}(\Omega)$. Indeed, since $u$ is radially decreasing,

$$
\|u\|_{L^{\infty}(B(0, R))}=u^{*}(0) \geq \int_{0}^{\omega_{n} R^{n}} f^{*}(t) t^{\frac{m}{n}-1} d t=\infty
$$

inasmuch as $f \notin L^{\frac{n}{m}, 1}(0,1)$.

The existence of a function $u \in W^{m} X_{\mathrm{loc}}(\Omega) \backslash L_{\mathrm{loc}}^{\infty}(\Omega)$ contradicts assumption (i), which forces $W^{m} X_{\text {loc }}(\Omega)$ to be contained in $L_{\text {loc }}^{\infty}(\Omega)$.

Acknowledgment. This research was partly supported by the PRIN research project "Geometric aspects of partial differential equations and related topics" (2008) of MIUR (Italian Ministry of University and Research), and by GNAMPA of the Italian INdAM (National Institute of High Mathematics).

We wish to thank the referees for their careful reading of the manuscript, and for their valuable comments.

\section{References}

[1] Aïssaoui, N.: Maximal operators, Lebesgue points and quasicontinuity in strongly nonlinear potential theory. - Acta Math. Univ. Comenian. (N.S.) 71:1, 2002, 35-50.

[2] Alberico, A., and A. Cianchi: Differentiability properties of Orlicz-Sobolev functions. Ark. Mat. 43:1, 2005, 1-28.

[3] Bennett, C., and R. Sharpley: Interpolation of operators. - Academic Press, Boston, 1988.

[4] Brezis, H., and S. WAINGER: A note on limiting cases of Sobolev embeddings and convolution inequalities. - Comm. Partial Differential Equations 5:7, 1980, 773-789.

[5] Cianchi, A.: Continuity properties of functions from Orlicz-Sobolev spaces and embedding theorems. - Ann. Sc. Norm. Super. Pisa Cl. Sci. (4) 23:3, 1996, 575-608.

[6] Cianchi, A.: Symmetrization and second-order Sobolev inequalities. - Ann. Mat. Pura Appl. (4) $183: 1,2004,45-77$.

[7] Cianchi, A.: Optimal Orlicz-Sobolev embeddings. - Rev. Mat. Iberoam. 20:2, 2004, 427-474.

[8] Cianchi, A.: Higher-order Sobolev and Poincaré inequalities in Orlicz spaces. - Forum Math. $18: 5,2006,745-767$.

[9] Cianchi, A., D. E. Edmunds, and P. Gurka: On weighted Poincaré inequalities. - Math. Nachr. 180, 1996, 15-41.

[10] Cianchi, A., and L. Pick: Sobolev embeddings into BMO, VMO, and $L^{\infty}$. - Ark. Mat. 36:2, $1998,317-340$. 
[11] Cianchi, A., L. PICK, and L. SlavíKová: Higher-order Sobolev embeddings and isoperimetric inequalities. - Preprint.

[12] Cianchi, A., and M. Randolfi: Differentiability properties of functions from higher-order Orlicz-Sobolev spaces. - Nonlinear Anal. 75:7, 2012, 3322-3338.

[13] Ciesielski, M., and A. Kaminska: Lebesgue's differentiation theorems in R.I. quasi-Banach spaces and Lorentz spaces $\Gamma_{p, w}$. - J. Funct. Spaces Appl. 2012, Art. ID 682960, 1-28.

[14] Curbera, G. P., and W. J. Rickel: Can optimal rearrangement invariant Sobolev imbeddings be further extended? - Indiana Univ. Math. J. 56:3, 2007, 1479-1497.

[15] Curbera, G. P., and W. J. Rickel: Sobolev type inequalities for rearrangement invariant spaces. - Positivity 15:4, 2011, 553-570.

[16] Cwikel, M., and E. Pustylnik: Sobolev-type embeddings in the limiting case. - J. Fourier Anal. Appl. 4:4-5, 1998, 433-446.

[17] DeVore, R.A., and R. C. Sharpley: On the differentiability of functions in $\mathbf{R}^{n}$. - Proc. Amer. Math. Soc. 91:2, 1984, 326-328.

[18] Edmunds, D.E., R. Kerman, and L. Pick: Optimal Sobolev imbeddings involving rearrangement-invariant quasinorms. - J. Funct. Anal. 170:2, 2000, 307-355.

[19] Fremlin, D. H.: Measure theory 3. - Torres Fremlin, Colchester, 2004.

[20] Kerman, R., and L. Pick: Optimal Sobolev imbeddings. - Forum Math. 18:4, 2006, 535-570.

[21] KLImov, V. S.: Imbedding theorems and geometric inequalities. - Izv. Akad. Nauk SSSR Ser. Mat. 40:3, 1976, 645-671, 710 (in Russian).

[22] LEvis, F. E.: Weak inequalities for maximal functions in Orlicz-Lorentz spaces and applications. - J. Approx. Theory 162:2, 2010, 239-251.

[23] MAZ'YA, V.: Sobolev spaces with applications to elliptic partial differential equations. Second, revised and augmented edition. - Springer, Heidelberg, 2011.

[24] O'Neil, R.: Convolution operators in $L(p, q)$ spaces. - Duke Math. J. 30, 1963, 129-142.

[25] Peetre, J.: Espaces d' interpolation et théorème de Soboleff. - Ann. Inst. Fourier 16:1, 1966, 279-317 (in French).

[26] PÉrez, C.: Two weighted inequalities for potential and fractional type maximal operators. Indiana Univ. Math. J. 43:2, 1994, 663-683.

[27] Stein, E. M.: Editor's note: The differentiability of functions in $\mathbf{R}^{n}$. - Ann. of Math. (2) $113: 2,1981,383-385$.

[28] Ziemer, W. P.: Weakly differentiable functions. - Springer-Verlag, New York, 1989.

Received 20 April 2013 • Accepted 17 December 2013 